\title{
Interaction of a monopole vortex with an isolated topographic feature in a three-layer geophysical flow
}

\author{
E. A. Ryzhov ${ }^{1}$ and K. V. Koshel ${ }^{1,2}$ \\ ${ }^{1}$ V.I.Il'ichev Pacific Oceanological Institute, 43, Baltiyskaya Street, Vladivostok, 690041, Russia \\ ${ }^{2}$ Far Eastern Federal University, 8, Sukhanova Street, Vladivostok, 690950, Russia
}

Correspondence to: E. A. Ryzhov (ryzhovea@poi.dvo.ru)

Received: 27 September 2012 - Revised: 5 December 2012 - Accepted: 10 January 2013 - Published: 14 February 2013

\begin{abstract}
In the frame of a three-layer, quasi-geostrophic analytical model of an $f$-plane geophysical flow, the Lagrangian advection induced by the interaction of a monopole vortex with an isolated topographic feature is addressed. Two different cases when the monopole is located either within the upper or the middle layer are of our interest. In the bottom layer, there is a delta-function topographic feature, which generates a closed recirculation region in its vicinity due to the background flow. This recirculation region extends to the middle and upper layers, and it plays the role of a topographic vortex. The interaction between the monopole and the topographic vortex causes a complex, including chaotic, advection of fluid particles. We show that the model's parameters, namely the monopole and topographic vortices' strengths and initial positions, and the layers' depths and densities, are responsible for the diverse advection patterns. While the patterns are rather complicated, one can single out two major processes, which mostly govern the fluid particle advection. The first one is the variation in time of the system's phase space structure, so that within the closed region of the topographic vortex, there appear periodically unclosed particle pathways by which the particles leave the topographic vortex. The second one is chaotic advection that arises from the nonstationarity of the monopole-topography interaction.
\end{abstract}

\section{Introduction}

Generally speaking, topographic vortices are coherent vortical structures appearing as closed recirculation regions over bottom features in the ocean and atmosphere. Topographic vortices play a fundamental role in the mass, salinity and temperature advection (transport) in the ocean. Moreover, topographic vortices are known to influence the dynamics of different coherent structures, such as unrestrictedly moving vortices (e.g. van Geffen and Davies, 1999; Dewar, 2002; Herbette et al., 2003; An and McDonald, 2005; Herbette et al., 2005; Sutyrin et al., 2011; Candon and Marshall, 2012; Zavala Sansón et al., 2012). Such topographic vortices greatly vary in vorticity and size scales in time (Baines and Smith, 1993; Baines, 1993). In this paper, however, we are only interested in meso- and synoptic-scale topographic vortices, as these scales are generally believed to be prevailing in the ocean (Chelton et al., 2007, 2011).

The present paper deals with the Lagrangian regular and irregular (chaotic) advection generated by a vortex monopole interacting with a topographic vortex. The topographic vortex under investigation is generated by a regular threelayer $f$-plane background flow (e.g. Pedlosky, 1987; Kozlov, 1995) with a delta-function bottom irregularity within the lower layer (e.g. Sokolovskiy et al., 1998; Izrailsky et al., 2004; Kozlov et al., 2005). Then we embed a monopole point-vortex (e.g. Gryanik, 1983; Gryanik and Tevs, 1989; Gryanik et al., 2000; Carton, 2001; Reznik, 2010; Reznik and Kizner, 2010) either within the upper or middle layer. So, these singularities move like passive tracers along regular background-flow streamlines (Reznik and Kizner, 2007a,b, 2010), while generating a complex - either periodic (for a time-independent background flow) or quasi-periodic (for a periodically time-dependent background flow) - velocity field in their own vicinity. Our main reason for employing such a three-layer model (e.g. Sokolovskiy, 1997; Ryzhov and Koshel, 2011a) is to study the Lagrangian advection induced by the monopole-topography interaction when the monopole is located within either the upper or middle layers. Such monopole positioning can be considered as the simplest 
models for a surface eddy and for an interthermocline lens (e.g. Carton et al., 2002; Wang and Dewar, 2003; Filyushkin et al., 2011; Filyushkin and Sokolovskiy, 2011), respectively. However, we should emphasize that the paper deals only with the surface Lagrangian advection in both cases. The middlelayer point-monopole appears in the upper layer as a regular vortex; thus, this configuration generates different fluid particle advections due to the absence of singularity in the monopole-topography velocity field.

We investigate two kinds of the monopole-topography interaction: the first one is an infinite-time interaction and the second one is a finite-time interaction. The infinite-time interaction means that the monopole moves in closed regular trajectories about the topographic vortex's elliptic point for infinite time due to the constancy of the background flow. However, if the background flow depends periodically on time, the dynamics of the monopole become more complicated. The monopole itself can be captured within the topographic vortex from the background flow or, conversely, be released from the topographic vortex into the background flow and consequently be carried away to infinity.

Thus, the main aim of the present study is to investigate the Lagrangian advection of fluid particles due to the velocity field generated by the infinite and short-term monopoletopography interactions.

\section{Equations of motion}

We study a three-layer QG flow model with the stream functions presented in Appendix A (see Eq. A6). In a dimensionless form, the model equations comprise important dimensionless quantities, which will be further used as parameters governing the different regimes of the Lagrangian advection. Introduce length scale $L=\left(k_{3}\left(\alpha_{2}-1\right)\right)^{-1 / 2}$; velocity scale $U$; the Rossby number, $\varepsilon=\frac{U}{f L}$; and an effective volume of the topography as $\tau=\pi h_{0} L^{2}$, where $h_{0}, L$ are the height and radius of a corresponding cylinder (Sokolovskiy et al., 1998). Then, the following governing parameters are

$\chi=\frac{f \tau}{H_{3} U L}=\frac{h_{0} \pi}{\varepsilon H_{3}}, \kappa_{m}=\frac{f \mu_{m}}{H_{m} U L}$,

which characterize the dimensionless topographic vortex strength and the dimensionless monopole strength, respectively. Then, by satisfying the QG flow requirement that $\frac{h_{0}}{H_{3}} \sim O(\varepsilon)$, we set $\chi=\pi$. Thus, choosing the following parameter values, $H_{1}=200 \mathrm{~m}, H_{2}=400 \mathrm{~m}, H_{3}=$ $3000 \mathrm{~m}, \rho_{1}=1026.56 \mathrm{~kg} \mathrm{~m}^{-3}, \rho_{2}=1027.84 \mathrm{~kg} \mathrm{~m}^{-3}$, and $\rho_{3}=1028.32 \mathrm{~kg} \mathrm{~m}^{-3}$, we obtain the characteristic horizontal topographic vortex scale $L \sim 1.3 \times 10^{4} \mathrm{~m}$.

Now, by making use of the dimensionless parameters and the geostrophic relations, one can write the equations of motion for the monopole's centre and for a fluid particle advected by the monopole-topography velocity field. The monopole motion in the $m=1,2$-layer is governed by the following equations of motion:

$$
\begin{aligned}
& \frac{d}{\mathrm{~d} t} x_{m}^{*}=-\left.\frac{\partial \psi_{m m}}{\partial y}\right|_{\substack{x=x_{m}^{*} \\
y=y_{m}^{*}}}=W+\chi \frac{y_{m}^{*}}{r_{m}^{*}} V_{m}\left(r_{m}^{*}\right), \\
& \frac{d}{\mathrm{~d} t} y_{m}^{*}=\left.\frac{\partial \psi_{m m}}{\partial x}\right|_{\substack{x=x_{m}^{*} \\
y=y_{m}^{*}}}=-\chi \frac{x_{m}^{*}}{r_{m}^{*}} V_{m}\left(r_{m}^{*}\right),
\end{aligned}
$$

where $W=W(t)$ is the dimensionless background flow velocity,

$$
\begin{aligned}
& V_{m}(\xi)=\frac{1}{\gamma}\left(\left(\alpha_{1} \beta_{2}-\alpha_{2} \beta_{1}\right) \frac{1}{\xi}+\alpha_{m}\left(\beta_{1}-\beta_{2}\right) K_{1}(\xi)+\right. \\
& \left.+\beta_{m}\left(\alpha_{2}-\alpha_{1}\right) \sqrt{\frac{\left(\beta_{2}-1\right)}{\left(\alpha_{2}-1\right)}} K_{1}\left(\sqrt{\frac{\left(\beta_{2}-1\right)}{\left(\alpha_{2}-1\right)}} \xi\right)\right),
\end{aligned}
$$

and $r_{m}^{*}=\sqrt{\left(x_{m}^{*}\right)^{2}+\left(y_{m}^{*}\right)^{2}}$ is the monopole position in the $m$-layer. System (2) is a thoroughly-studied system which governs the dynamics of a fluid particle due to the velocity field generated by an exterior background flow intersecting a delta-function topography. The Lagrangian advection induced by system (2) has been studied recently in the frame of barotropic (Sokolovskiy et al., 1998; Izrailsky et al., 2004; Koshel and Prants, 2006), two-layer (Kozlov et al., 2005; Ryzhov and Koshel, 2011b) and three-layer baroclinic geophysical flows (Ryzhov and Koshel, 2011a). In our case, however, system (2) governs not the fluid particle dynamics, but the monopole-centre dynamics. Thus, the upperand middle-layer monopole move as a fluid particle due to the topographic vortex velocity field. Fluid particles of the monopole-topography interaction system, however, undergo the joint influence of both the monopole and topographic vortex velocity fields.

Motion of a fluid particle influenced by the cooperative monopole-topography velocity field obeys the relations

$$
\begin{aligned}
\dot{x}_{i} & =-\frac{\partial \psi_{i m}}{\partial y_{i}}= \\
& =W+\kappa_{m} \frac{\left(y_{i}-y_{m}^{*}\right)}{r_{i m}^{*}} P_{i m}\left(r_{i m}^{*}\right)+\chi \frac{y_{i}}{r_{i}} V_{i}\left(r_{i}\right), \\
\dot{y}_{i} & =\frac{\partial \psi_{i m}}{\partial x_{i}}= \\
& =-\left(\kappa_{m} \frac{\left(x_{i}-x_{m}^{*}\right)}{r_{i m}^{*}} P_{i m}\left(r_{i m}^{*}\right)+\chi \frac{x_{i}}{r_{i}} V_{i}\left(r_{i}\right)\right),
\end{aligned}
$$

where $r_{i m}^{*}=\sqrt{\left(x_{i}-x_{m}^{*}\right)^{2}+\left(y_{i}-y_{m}^{*}\right)^{2}}$ is the fluid particle position relative to the monopole's centre position,

$$
\begin{gathered}
P_{i m}(\xi)=\frac{(-1)^{3-m}}{\gamma}\left(\left(\alpha_{3-m}-\beta_{3-m}\right) \frac{1}{\xi}+\alpha_{i}\left(\beta_{3-m}-1\right)\right. \\
\left.K_{1}(\xi)+\beta_{i}\left(1-\alpha_{3-m}\right) \sqrt{\frac{\left(\beta_{2}-1\right)}{\left(\alpha_{2}-1\right)}} K_{1}\left(\sqrt{\frac{\left(\beta_{2}-1\right)}{\left(\alpha_{2}-1\right)}} \xi\right)\right),
\end{gathered}
$$

and $m=1,2$ corresponds to the upper- and middle-layer monopole cases. 


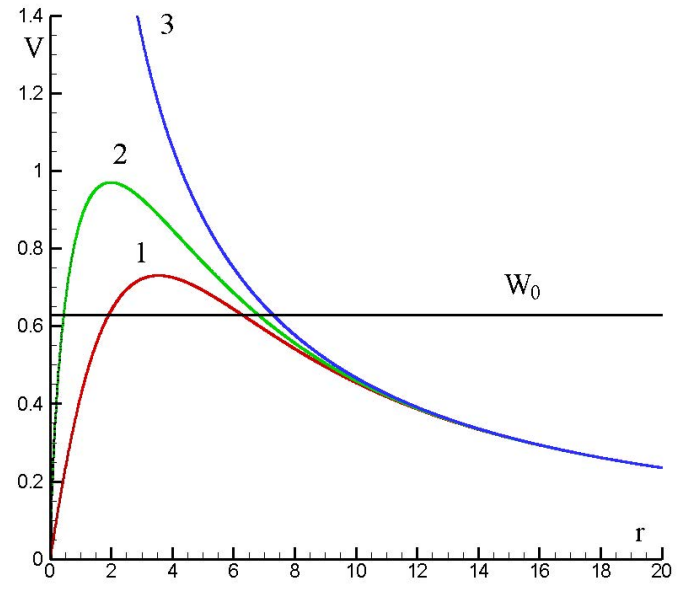

Fig. 1. Azimuthal velocities of the topographic vortex within the layers. Curves 1,2, 3 correspond to the upper, middle, and bottom layer, respectively. The horizontal straight line indicates constant background velocity value $W_{0}=0.2 \pi$.

\section{Monopole motion}

First, we briefly analyse system (2), which governs the monopole's dynamics. An elaborated study of this system has been conducted (Ryzhov and Koshel, 2011a). If the background exterior flow is constant ( $\left.W=W_{0}\right)$, system (2) is integrable in the sense of the streamline-trajectory coincidence (e.g. Zaslavsky, 1998). Because the bottom topography is singular, any nonzero value of $W_{0}$ always produces a closed Taylor column region called a topographic vortex within the lower layer. To have such closed regions within the middle and upper layers, however, the background velocity should be lower than a critical value. This critical value is the maximal value of the azimuthal velocity in the corresponding layer. So, if one chooses the background flow to satisfy this condition, then three different-sized Taylor columns will occur due to the bottom irregularity. These three columns may be thought of as a discrete Taylor cone. Figure 1 depicts azimuthal velocities $V_{i}$ depending on distance $r$ to the topographic vortex elliptic point. We chose $W_{0}=0.2 \pi, \chi=\pi$ to ensure the mesoscale closed regions exist in all three layers. The points, where the horizontal line intersects the azimuthal velocity curves, correspond to elliptic and hyperbolic critical points of the vortex. Figure 2 demonstrates streamlines of the resulting topographic vortex in the upper layer. The red curve indicates the separatrix dividing the flow into the vortical region and the exterior flow. Since we also are interested in the middle-layer monopole case, the vortical region of the middle layer is indicated by the blue dashed curve.

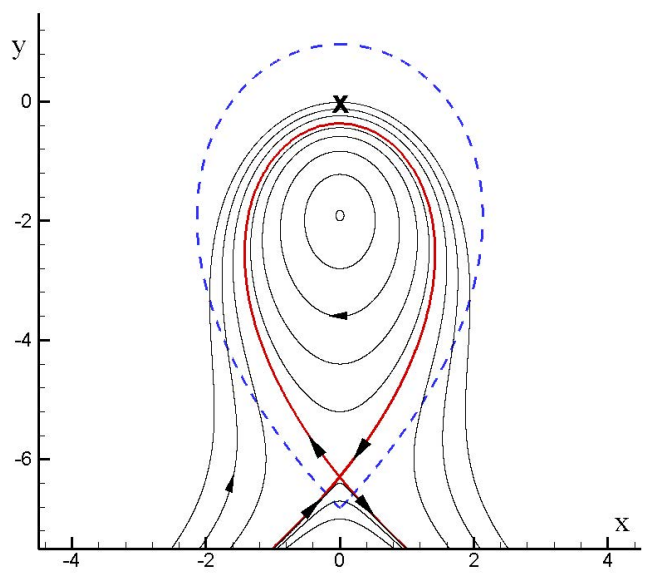

Fig. 2. Topographic vortex streamlines for the upper layer. The red curve indicates the separatrix. The dashed blue curve corresponds to the middle-layer separatrix. The cross marks the delta-function topography position, $(0,0)$.

\section{Fluid particle advection}

\subsection{Regular monopole motion}

Now we can analyse the Lagrangian advection induced by the monopole-topography interaction velocity field. Motion of a fluid particle is governed by system (3), where the righthand part of the relations comprises the monopole motion solution given by Eq. (2). First, we consider the periodic solution of Eq. (2). This solution, although it cannot be expressed in an analytical form, is time-dependent with a period being equal to the time of the monopole passing a closed trajectory within the separatrices shown in Fig. 2. Hence, system (3) is a dynamical system with one-and-a-half degrees of freedom that allows the irregular dynamics of the fluid particle, conventionally called chaotic advection (Aref, 1984, 2002; Wiggins, 1992). Chaotic advection manifests itself through exponential divergence of close trajectories in a finite time (e.g. Lichtenberg and Lieberman, 1983; Zaslavsky, 1998). The easiest way to demonstrate the chaotic advection manifestation is by constructing Poincaré sections of system (3). Figure 3a shows a Poincaré section as $\kappa_{1}=0.01, y_{1}^{*}(0)=-4$, corresponding to frequency $\omega=0.1611$ of monopole rotation along an orbit shown in Fig. 2.

That half degree of freedom corresponds to a timedependent perturbation, which concerning system (3) is the monopole motion term comprising strength $\kappa_{m}$. However, this monopole strength is not the only parameter greatly affecting the Lagrangian advection; the initial position of the monopole is also of great importance. Indeed, initial positions of the monopole determine the orbit the monopole will pass along, and different orbits correspond to different rotational frequencies. These, in turn, are the frequencies of the periodic perturbation that are introduced by the monopole 

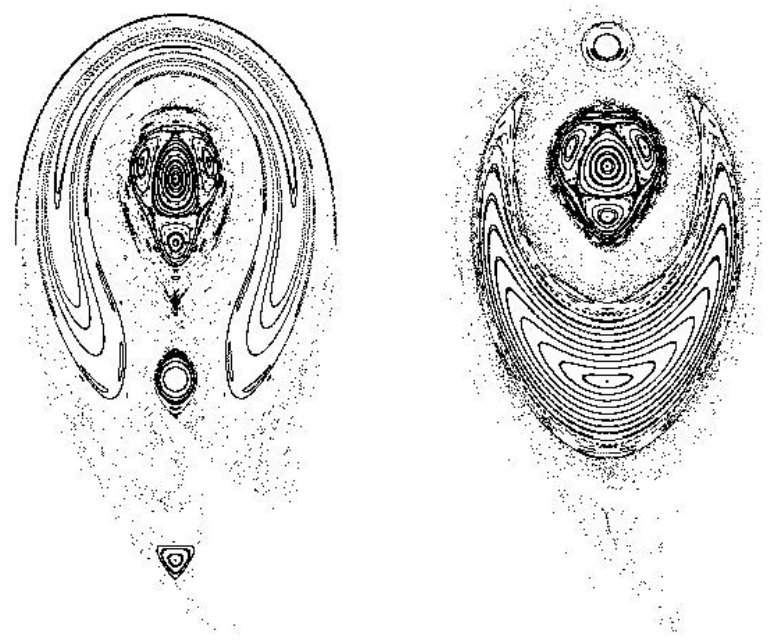

(a) $(0,-4)$

(b) $(0,-0.6936)$

Fig. 3. Equivalent Poincaré sections of system (3) for the same values of $\kappa=0.01$ and the same values of perturbation frequency $\omega$ but different monopole initial positions $\left(x_{1}^{*}, y_{1}^{*}\right)$.

into the topographic vortex region. As the initial position parameter, we choose the positions where the streamlines (shown in Fig. 2) intersect the y-axis. Figure 3a and b show the equivalent structures of system (3) phase space for different initial positions of the monopole, corresponding nevertheless to the same streamline. Hence, the positions on the $y$-axis correspond to all the frequencies of the monopole rotation about topography. Thus, we will further address how the monopole's strength and initial position parameters affect the fluid particle dynamics.

The Poincaré section analysis is a very useful technique to estimate which part of fluid particles is involved either in regular advection or in chaotic advection; however, this technique fails to show what happens with fluid particles at certain moments. So, to address the question of how these fluid particles move while the monopole achieves a revolution about the topography, we calculate the number of critical points that appear at each instant in the flow (Ryzhov and Koshel, 2011b; Ryzhov et al., 2012). The simple idea of this classification is that the more critical points of their initial set survive or, in other words, the fewer topological changes appear during a monopole revolution, the more regular system (3) is.

\subsection{Diagram of the number of the critical points}

As the monopole moves about topography, the number of the regular critical points changes, altering flow topology characteristics with time (e.g. Aref and Brons, 1998). It should

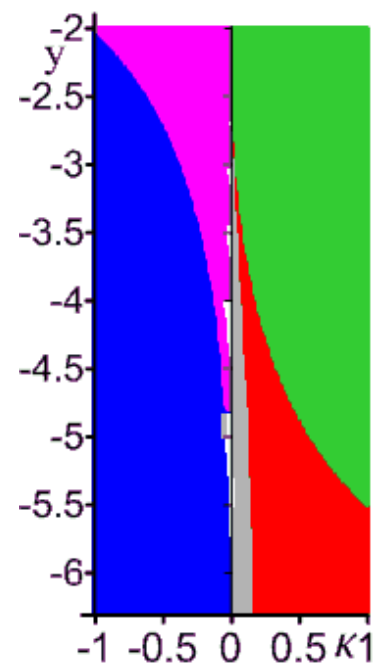

(a) upper-layer monopole case

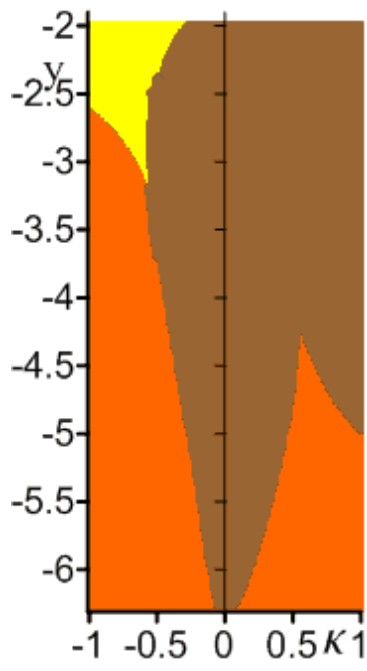

(b) middle-layer monopole case
Fig. 4. (a) and (b) correspond to the upper-layer monopole case and to the middle-layer monopole case, respectively. Number of the flow's regular critical points by colour. Blue -3 initial points and 3 or 5 points at half period; purple -5 initial points and 5 points at half period; grey -3 initial points and 3 points at half period; red 3 initial points and 1 point at half period; and green -1 initial point and 1 point at half period. Yellow -6 initial points and 2 points at half period; orange -4 initial points and 2 points at half period; and brown -2 initial points and 2 points at half period.

be mentioned that, in the upper-layer monopole case, one singular critical point corresponding to the monopole's centre always exists, so we have excluded it from consideration. In the middle-layer monopole case, no singular points occur within the upper-layer velocity field because the middle-layer point-monopole appears as a regular one in the upper layer. So, making use of the classification based on the number of regular critical points appearing at certain instants of time, we present diagrams of the number of the regular critical points in the upper-layer monopole case and in the middlelayer monopole case, respectively, depending on monopole's strength $\kappa$ and initial position $y$. These diagrams depict by colour how many regular critical points appear at the beginning of the monopole rotation (initial critical points) and at the time the monopole has passed half a rotation period (critical points at half period).

In these diagrams, the $\kappa<0$ region corresponds to counter-rotation of the monopole and topographic vortex, and $\kappa>0$ corresponds to corotation of the monopole and topographic vortex. First, we consider the upper-layer monopole case. Figure 5 shows the flow's streamlines at the initial stage of monopole motion and the stage at half period. The red curves are the monopole trajectories, and the dashed blue curve corresponds to the unperturbed topographic vortex separatrix. 
Also, as a Lagrangian advection measure, we have calculated the escape time (Kozlov and Koshel, 1999, 2000; Izrailsky et al., 2004), which is the time a fluid particle needs to be carried away by the exterior flow from the topographic vortex region. This measure is an analogue to the Lyapunov exponent, and it shows where the Lagrangian advection progresses faster or slower. Thus, we have uniformly distributed within the separatrix $10^{4}$ markers and then taken into consideration the time they would need to cross the line far enough out of the vortex interaction (we chose line $x=5$ ). The escape-time distributions are shown in Fig. 8, where unity of the time is equal to the corresponding period of a monopole revolution. A general feature of all the subfigures is the almost circular areas of long-lived fluid particles. These areas correspond to the monopole region with a very high velocity that tends to infinity, approaching the monopole's centre. Hence, fluid particles within these areas move regularly (Ryzhov and Koshel, 2011b), and, therefore, they do not leave the topographic vortex region.

The blue region corresponds to a strong influence of the monopole motion. At the initial stage, there are three regular critical points that form a heteroclinic structure (see Fig. 5a). The topographic vortex cannot be distinctly identified because no instantaneous hyperbolic point corresponds to the unperturbed hyperbolic point. So, this case of monopoletopography interaction cannot be considered as a perturbation of the topographic vortex. Moreover, this initial streamline pattern resembles a counter-rotating dipole structure (e.g. Voropayev et al., 2001; Ryzhov, 2011). The change with time of this structure produces, at half period, three regular critical points forming two homoclinic structures associated either with the topographic or monopole vortices (see Fig. 5b). Due to that topological alteration, the Lagrangian advection is very effective. Most particles are carried away within 5 monopole revolutions (see Fig. 8a).

The purple region corresponds to a moderate influence of the monopole motion. At the initial stage, five regular critical points form one heteroclinic and one homoclinic structure (see Fig. 5c). This homoclinic structure almost coincides with the unperturbed topographic vortex separatrix, which indicates that this case can be considered as a perturbation of the topographic vortex flow. At half period, the streamline pattern appears almost the same as before in the blue region (see Fig. 5d). The Lagrangian advection still occurs fast, and extends over the whole separatrix region, but is less efficient than before. No stagnation zone appears within the region (see Fig. 8b).

The grey region corresponds to the weakest monopole influence. This positive $\kappa$ region differs topologically from those presented. Because the vortices are corotating, the initial topological structure appears as a corotating dipole enveloped by a common separatrix (see Fig. 5e). So, this structure can be considered as a topographic vortex with a double centre. Although this double centre greatly perturbs the fluid particle dynamics, the structure of the topographic vortex can be revealed during a whole monopole revolution (see Fig. 5f). Since there are two centres, fluid particles in the vicinity of the topographic vortex centre move almost regularly; however, the surrounding fluid is carried away very fast (see Fig. 8c).

The red region corresponds to a transitional case of the monopole-topography interaction. Initially, the streamline pattern appears as a corotating dipole structure (see Fig. 5g); then, during a monopole revolution, the dipole structure breaks, so that the point-monopole absorbs the topographic vortex elliptic point and becomes a new centre of the topographic vortex over a certain time. During this time span, the Lagrangian advection within the topographic vortex (with the new singular centre) is rather regular (see Fig. 5h). However, during a whole monopole revolution, almost all the fluid from the topographic vortex is carried away (see Fig. 8d).

The green region corresponds to the capture of the monopole, with the point-monopole centre playing the role of a new topographic vortex centre. Both at the initial stage and the stage at half period, the streamline patterns comprise only one regular critical point that corresponds to the hyperbolic point of the topographic vortex. The initial streamline pattern is shown in Fig. 5i, while the streamline pattern at half period appears as almost the same as that shown in Fig. 5j. Thus, this case can be thought of as the topographic trapping of a monopole vortex. The Lagrangian advection, in this case, differs insignificantly from the case previously addressed (see Fig. 8e).

Figures 6 and 7 depict two examples of particle scattering due to counter- and corotation of the monopole and topographic vortex. The dashed blue curve corresponds to the unperturbed topographic vortex separatrix and serves as the boundary for the initial distribution of $\sim 10^{4}$ markers. The bold curve is the trajectory of the monopole, with a triangle at the monopole's current position. Figure $6 \mathrm{c}$ shows how a prominent dipole structure appears due to the interaction. Comparing Figs. 6 and 7, one can see that the counterrotation case causes a more effective Lagrangian transport. However, the difference is rather small.

Now, we consider the diagram shown in Fig. $4 b$ for the middle-layer monopole case. The main difference from the upper-layer monopole case is that no singular point appears in the upper-layer velocity field; thus, the monopole appears regular and the topographic vortex can also be broken. Hence, a merger of the vortices can appear because both vortices are regular. This regularity leads all the streamline patterns at half period to appear almost the same with one elliptic point, which is formed by the merger, and one hyperbolic point. Also, the lack of singular points leads to a much more regular Lagrangian advection than above.

The yellow region corresponds to six initial regular critical points (see Fig. 9a) and two critical points at half period (see Fig. 9b), which both correspond to the topographic vortex with the monopole vortex having disappeared since the velocity of surrounding flow is too high for a closed 


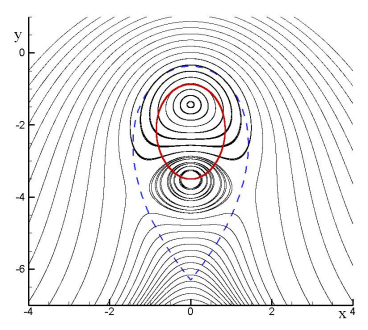

(a) initial stage for the blue region $(\kappa=-0.5, y=-3.5)$

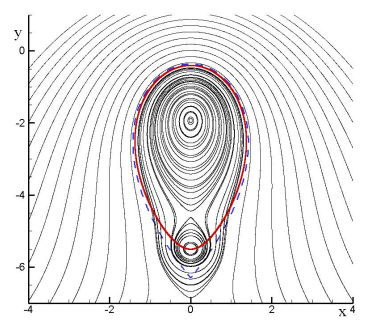

(e) initial stage for the grey region $(\kappa=0.1, y=-5.5)$

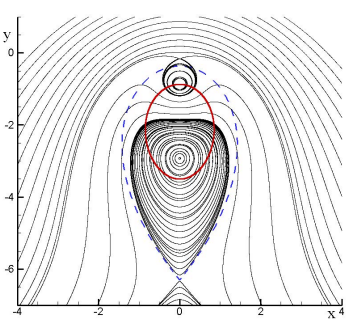

(b) stage at half period for the blue region $(\kappa=-0.5, y=-3.5)$

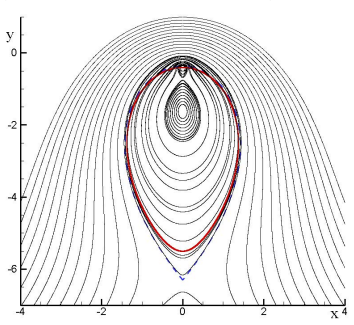

(f) stage at half period for the grey region $(\kappa=0.1, y=-5.5)$

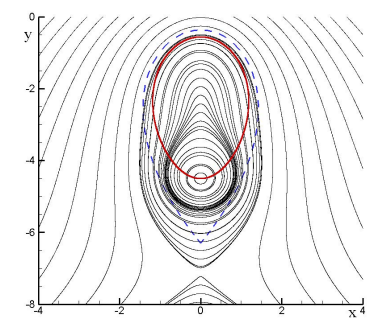

(i) initial stage for the green region $(\kappa=0.5, y=-4.5)$

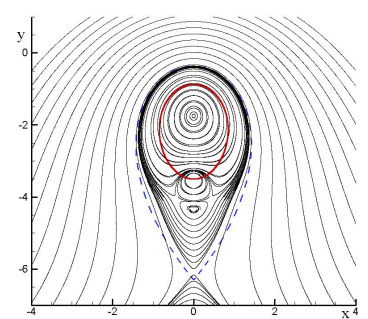

(c) initial stage for the purple region $(\kappa=-0.1, y=-3.5)$

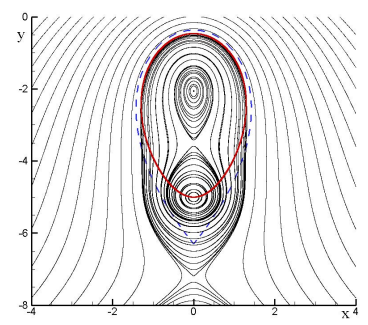

(g) initial stage for the red region $(\kappa=0.3, y=-5)$

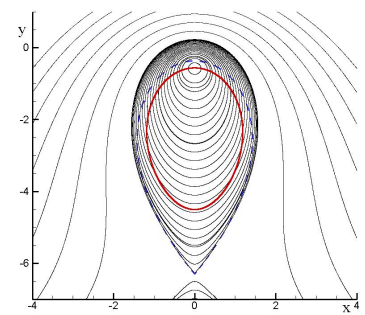

(j) stage at half period for the green region $(\kappa=0.5, y=-4.5)$

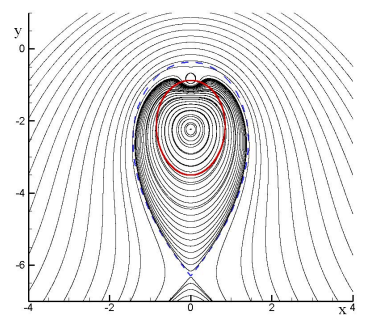

(d) stage at half period for the purple region $(\kappa=-0.1, y=-3.5)$

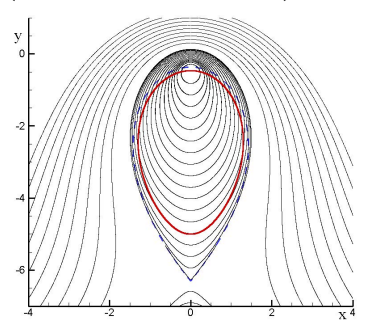

(h) stage at half period for the red region $(\kappa=0.3, y=-5)$

Fig. 5. Streamlines of the flow in the upper-layer monopole case. Red curve corresponds to the monopole motion trajectory. Dashed blue curve is the topographic vortex unperturbed separatrix.

circulation region to be formed. The position of the corresponding middle-layer monopole vortex is marked by a cross. Such a streamline pattern at half period is universal for all coloured regions shown in Fig. 9b. The corresponding escape-time distribution is shown in Fig. 10a. There is a big stagnation region with mostly regular advection corresponding to the lower closed region shown in Fig. 9a.

The orange region is arranged astride the $\kappa=0$ line. This region corresponds to the existence of three initial critical points. The difference between the negative and positive orange region initial streamline patterns is shown in Fig. 9c and d. Both of the corresponding streamline patterns at half period, however, appear as almost the same as that shown in Fig. 9b. Since initially two vortex structures can be reliably identified, and at half period these structures merge, the escape-time distribution shows a very effective and intense advection with no stagnation regions progressing. Figures 10c and d depict the escape-time distribution in the negative and positive $\kappa$ cases, respectively.

The brown region corresponds to the existence of two initial critical points and two critical points at half period. The middle-layer monopole does not induce a closed region within the upper layer. Despite that, the middle-layer monopole does greatly perturb the Lagrangian advection. The corresponding streamline pattern does not change topologically during a monopole revolution, and it appears as almost the same as that shown in Fig. 9b. However, on both sides of the $\kappa=0$ line, the advection efficiency is very different. In the $\kappa<0$ zone, the Lagrangian advection is very irregular (see Fig. 10d) due to counter-rotation of the middle-layer monopole and the topographic vortex. On the other hand, in the $\kappa>0$ zone, the Lagrangian advection is mostly regular, 


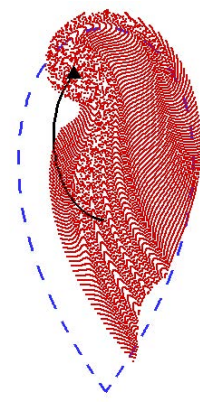

(a) $t=15$

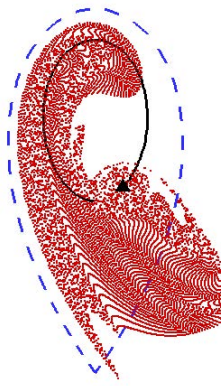

(b) $t=30$

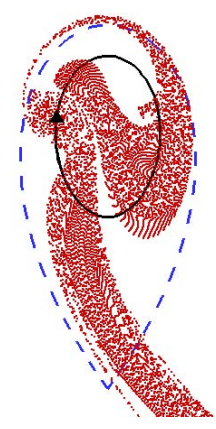

(c) $t=45$

Fig. 6. An example of particle scattering in the upper-layer monopole case due to counter-rotation of the monopole and topographic vortex, $\kappa=-0.1, y_{0}=-3.5$. The bold black curve is the trajectory of the monopole, with a triangle that shows the monopole's current position.

and a big stagnation region appears near the topographic vortex (see Fig. 10e), due to corotation of the middle-layer monopole and the topographic vortex.

We will further study how the irregular motion of the monopole influences the Lagrangian advection.

\subsection{Irregular monopole motion}

In this paragraph, we analyse the Lagrangian advection induced by an aperiodic perturbation consisting of a periodic background flow oscillation and a non-periodic part due to monopole irregular motion within the topographic vortex. This irregular monopole motion occurs because the pointmonopole's centre moves as a fluid particle in the periodically driven velocity field of the topographic vortex, which is known to produce the irregular dynamics (e.g. Sokolovskiy et al., 1998; Kozlov and Koshel, 2001; Izrailsky et al., 2004, 2008; Koshel et al., 2008). The aperiodic perturbation is of interest in the view of studying real geophysical flows, which are aperiodic by nature. Considerable progress has been made in this way due to the implementation of dynamical system theory (see a few recent studies: Mancho et al., 2006; Branicki and Wiggins, 2010; Mendoza et al., 2010; Rypina et al., 2011; Titaud et al., 2011). Strictly speaking, if one set the background flow to oscillate periodically,

$W=W_{0}\left(1+\mu_{W} \cos \nu_{W} t\right)$,

where $\mu_{W}$ and $\nu_{W}$ are the magnitude and frequency of the background flow oscillation, then system (2) becomes a system with one-and-a-half degrees of freedom permitting the chaotic dynamics to occur. Hence, with such an oscillating background flow, the monopole can start moving out of the topographic vortex, and then it can be trapped temporarily by the topography. And, conversely, if the monopole starts moving within the topographic vortex, it can be carried away by
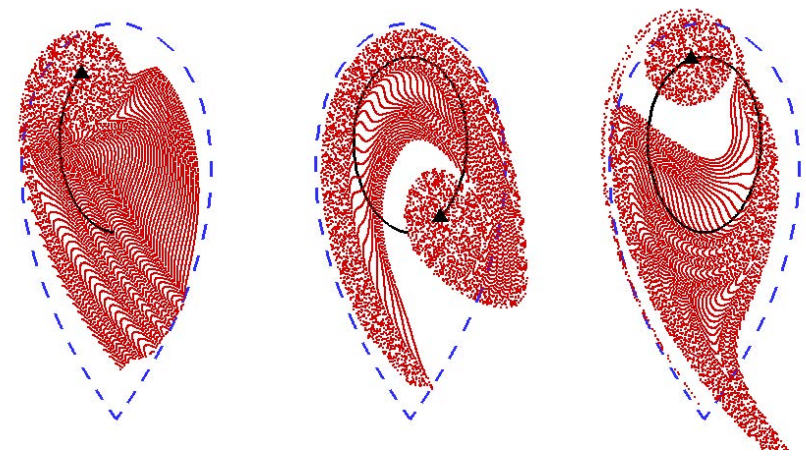

(a) $t=15$

(b) $t=30$

(c) $t=46$

Fig. 7. The same as in Fig. 6 due to corotation of the monopole and topographic vortex, $\kappa=0.1, y_{0}=-3.5$.

the exterior flow. It should be mentioned that the background flow oscillation also affects the fluid particle dynamics, resulting in a certain number of particles to leaving the topographic vortex region. However, in our numerical simulation, we chose a very small perturbation magnitude $\left(\mu_{W}=0.01\right)$, so there are very few such particles. So, by making use of such a configuration, we study the Lagrangian advection that is mostly induced by the short-term monopole-topography interaction.

Figure 11 depicts an example of the Lagrangian advection generated by the short-term interaction while the monopole accomplishes a few revolutions within the topographic vortex. Figure 11a shows the initial configuration of red and green markers corresponding to the topographic and monopole vortex regions, respectively. The unperturbed topographic vortex region is uniformly filled in with $10^{4}$ red markers. Also, $1.5 \times 10^{3}$ green markers are placed to distinguish the monopole vortex region. The monopole with strength $\kappa=0.1$ starts moving out of the topographic vortex (see Fig. 11a) at the point $x=-2, y=-8.4$. Then, the monopole vortex is captured by the topographic vortex due to chaotic advection (see Fig. 11b). Next, Fig. 11c shows the marker distribution as the monopole has passed half a rotational period (the black curve points out the trajectory of the monopole's centre). A great deformation caused by the monopole is clearly seen. Figure 11d illustrates the particle distribution after the monopole has made three whole revolutions about the topography. A few red markers from the initial distribution have stayed within the topographic region. Figure 11e depicts the monopole leaving the topographic vortex region after four revolutions.

Figure 12 also depicts a series of marker-scattering patterns, but for the middle-layer monopole case. In this case, the monopole starts moving at the position with coordinates $x=-1.18, y=-8$ and it appears as a regular vortex within the upper layer. As a consequence, a closed recirculation region corresponding to the monopole ceases to exist 


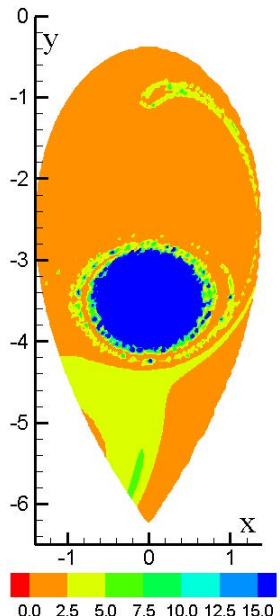

(a) $\kappa=-0.5$, $y=-3.5$

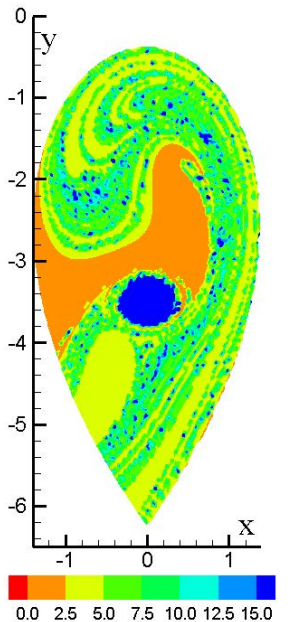

(b) $\kappa=-0.1$, $y=-3.5$

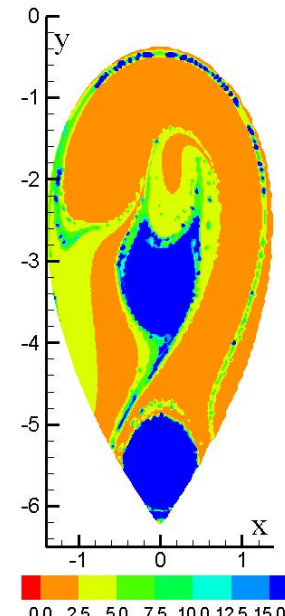

(c) $\kappa=0.1$, $y=-5.5$

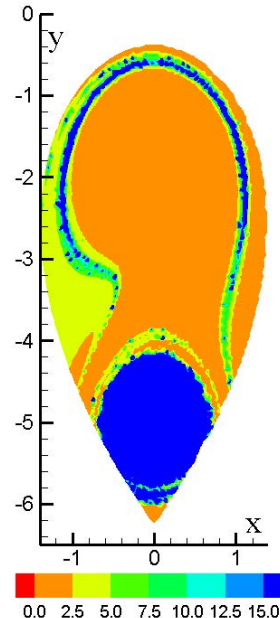

(d) $\kappa=0.3$, $y=-5$

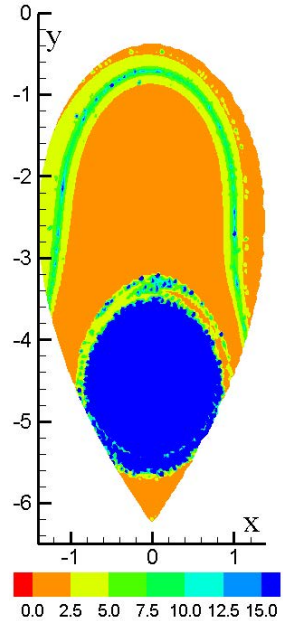

(e) $\kappa=0.5$, $y=-4.5$

Fig. 8. Escape-time distribution in the upper-layer monopole case.

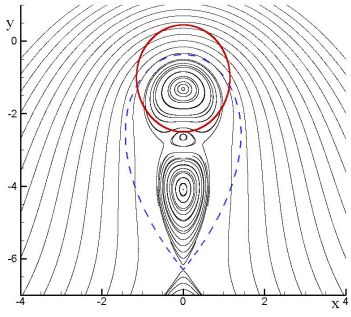

(a) initial stage for the yellow region $(\kappa=-0.7, y=-2.5)$

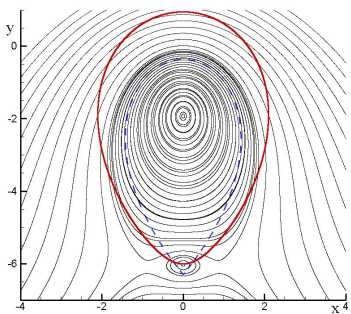

(c) initial stage for the negative orange region $(\kappa=-0.5, y=-6)$

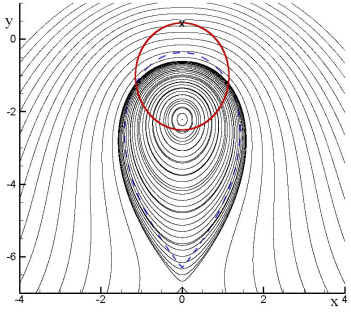

(b) stage at half period for the yellow region $(\kappa=-0.7, y=-2.5)$

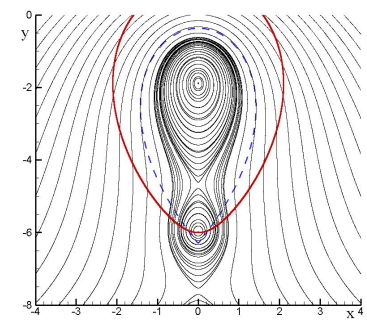

(d) initial stage for the positive orange region $(\kappa=0.5, y=-6)$
Fig. 9. Streamlines of the flow in the middle-layer monopole case. The red curve corresponds to the monopole motion trajectory. The dashed blue curve is the topographic vortex unperturbed separatrix.

at the stage at half period. Hence, the green markers mostly leave the monopole region (see Fig. 12c). However, when the closed recirculation region appears again (see Fig. 12d), the monopole captures a great deal of the red markers initially associated with the topographic vortex. Thus, during topography capture, the monopole encloses some red markers, then, after being carried away from the topographic vortex, it advects them to infinity (see Fig. 12e).

Figures 11 and 12 also clearly show that particle advection is greatly affected by the number of monopole revolutions about the topography. The longer the monopole revolves about the topography, the more effective advection is. To estimate that short-term monopole influence, we have performed a numerical simulation in which we calculated the number of fluid particles escaping the topographic vortex with respect to the number of the monopole revolutions. Since the monopole motion is irregular, two initially close monopole trajectories wind around the topography very differently, with different revolution numbers. Hence, it is impossible to predict how many revolutions the monopole will complete starting at a new initial position. Thus, as initial positions for the monopole, we have chosen two intervals of initial positions: $(x=-2, y \in[-8.42 ;-8.38])$ for the upperlayer monopole case and $(x=-1.18, y \in[-8.02 ;-7.98])$ for the middle-layer monopole case.

Then we followed the evolution of all the monopoles starting at these initial positions, calculating the revolution number, $N$, of each of those monopoles, and obtained the advection efficiency, $E=n_{\mathrm{a}} / n_{i}$, where $n_{\mathrm{a}}$ is the number of markers advected out of the topographic vortex. These are the markers that have crossed line $x=5$, and $n_{i}=10^{4}$ is the initial marker distribution number. It is also worth noting that, although some of these monopoles have revolved about the topography an equal number of times, the Lagrangian advection generated by these monopoles is mostly equivalent in 


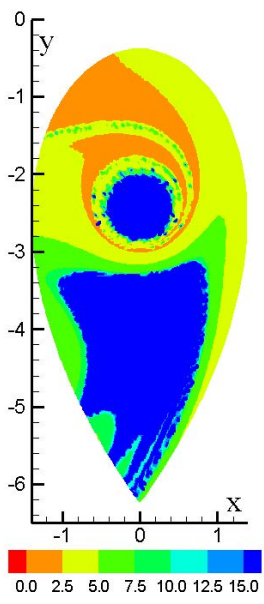
(a) $\kappa=-0.7$
$y=-2.5$

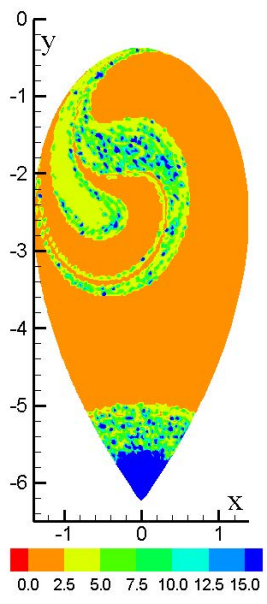

(b) $\kappa=-0.5$, $y=-6$

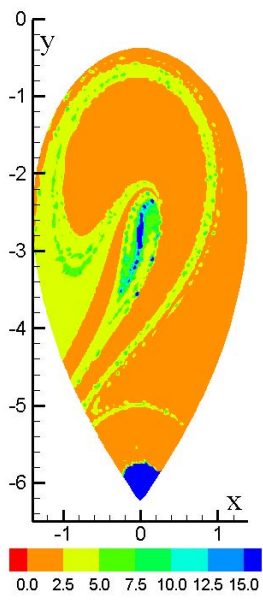

(c) $\kappa=0.5$, $y=-6$

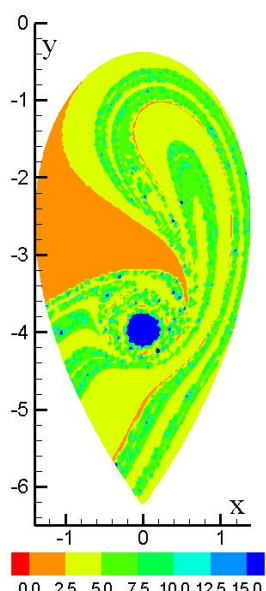

(d) $\kappa=-0.2$, $y=-4$

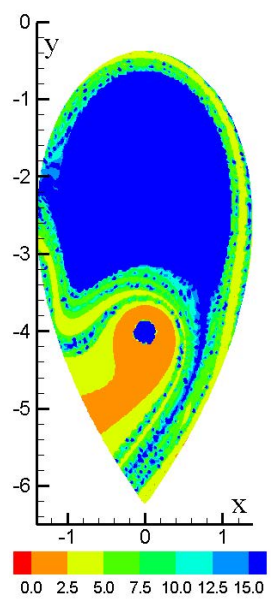

(e) $\kappa=0.2$, $y=-4$

Fig. 10. Escape-time distribution in the middle-layer monopole case.

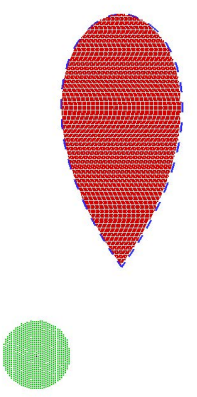

(a) $t=0$

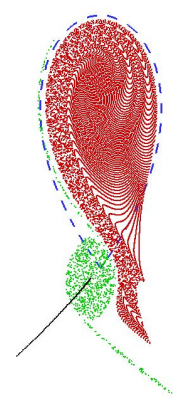

(b) $t=30$

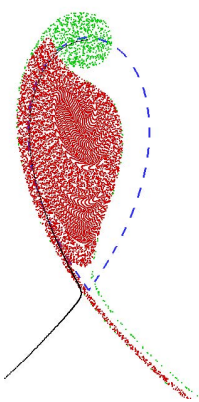

(c) $t=90$

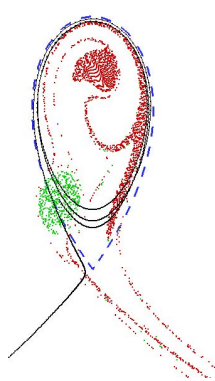

(d) $t=240$

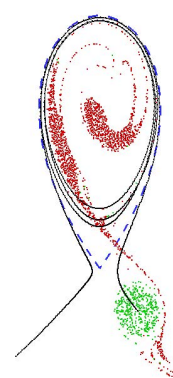

(e) $t=315$

Fig. 11. Upper-layer monopole case. Particle scattering at the short-term monopole-topography interaction. Red and green markers correspond to the topographic and monopole vortex regions, respectively. The blue dashed curve is the unperturbed topographic vortex separatrix, and the black curve points out the trajectory of the monopole's centre. Subfigures depict markers' distribution at the corresponding instant in time.

each case (see Fig. 13). Indeed, each point in Fig. 13 corresponds to one initial position of the monopole. Thus, if different initial positions correspond to an equal number of monopole revolutions $N$, then advection efficiency $E$ is sufficiently similar.

Figure 13 depicts advection efficiency $E$ in the upper-layer monopole case (see Fig. 13a and b) and in the middle-layer monopole case (see Fig. 13c and d). By analysing these subfigures, one can draw several conclusions. First, $N=0.5$ corresponds to the case of a monopole passing very close to the topographic vortex but not being captured by it. In this case, however, if a monopole is very weak $(\kappa=0.01)$, it causes a great deal of the fluid particle advection. A few monopole revolutions are enough for all the particles from the topographic vortex region to be carried away. Second, the sign of the monopole self-rotation is not the main reason for the advection efficiency, but this efficiency is mostly determined by $|\kappa|$ ). Third, evidently, a point-monopole (see Fig. 13a and b) causes a much more efficient advection than a regular one (see Fig. 13c and d).

\section{Conclusions}

In the frame of a three-layer geophysical flow model, the Lagrangian advection of fluid particle in the vicinity of a monopole vortex interacting with a topographic vortex has been addressed. Two cases of monopole propagation have been investigated: upper-layer monopole propagation and middle-layer monopole propagation. Such advection has been shown to be determined by two most significant processes: first, chaotic advection due to the nonstationarity of the monopole-topography interaction; and, second, the 


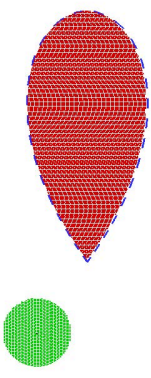

(a) $t=0$

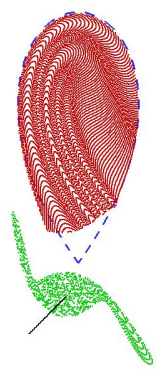

(b) $t=18$

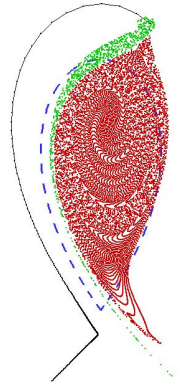

(c) $t=96$

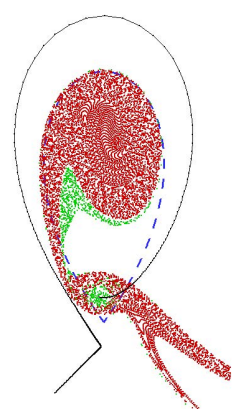

(d) $t=120$

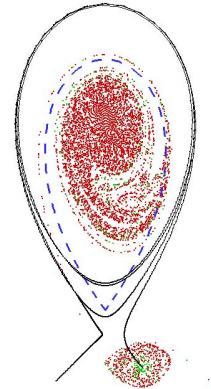

(e) $t=315$

Fig. 12. The same as in Fig. 11 for the middle-layer monopole case.

appearance or disappearance of closed recirculation zones in time. Cooperative influence of these processes causes a very efficient Lagrangian advection. Two controlling parameters, namely the monopole's strength and initial position, have been analysed, and, on the basis of the number of regular critical points assessment, a classification of different regimes of the Lagrangian advection has been presented.

By adding a nonstationary term to the background flow, we have analysed a short-term monopole-topography interaction. If the monopole passes near the topographic vortex, it causes a great deal of particles initially located within the topographic vortex to be carried away. If the monopole is captured by the topographic vortex, then it rotates a certain time about the topography and, finally, is carried away by the background flow. During this passage, the topographic vortex almost completely renews its fluid.

\section{Appendix A}

\section{Three-layer QG flow}

For a background three-layer QG flow under the rigid-lid approximation, the potential vorticity, $q_{i}$, in the $i$-layer, where $i=1,2,3$ corresponds to the upper, middle, and lower layer, is equal to (Pedlosky, 1987)

$q_{1}=\Delta \psi_{1}+\frac{f}{H_{1}} \zeta_{1}+f, q_{2}=\Delta \psi_{2}+\frac{f}{H_{2}}\left(\zeta_{2}-\zeta_{1}\right)+f$,

$q_{3}=\Delta \psi_{3}+\frac{f}{H_{3}}\left(h(x, y)-\zeta_{2}\right)+f$,

where $\Delta \psi_{i}=\frac{\partial v_{i}}{\partial x}-\frac{\partial u_{i}}{\partial y}$ is the 2-D relative vorticity with stream function $\psi_{i}$ and two-dimensional velocity field $u_{i}, v_{i}$; $\zeta_{1}, \zeta_{2}$ are the interface heights between the upper and middle, and the middle and lower layers; $h(x, y)=\tau \delta(\boldsymbol{r}))$ is the Dirac delta-function bottom irregularity with effective volume $\tau ; H_{i}$ is the $i$-layer depth; and $f$ is the constant Coriolis parameter. According to the pressure continuity condition, the interface heights can be written in the form (Pedlosky, 1987)

$\zeta_{1}=\frac{f\left(\psi_{2}-\psi_{1}\right) \rho_{2}}{\left(g\left(\rho_{2}-\rho_{1}\right)\right)}, \zeta_{2}=\frac{f\left(\psi_{3}-\psi_{2}\right) \rho_{3}}{\left(g\left(\rho_{3}-\rho_{2}\right)\right)}$,

where $\rho_{i}$ is the $i$-layer fluid density; $g$ is the gravitational acceleration; and $\Delta \rho_{1}=\rho_{2}-\rho_{1}$ and $\Delta \rho_{2}=\rho_{3}-\rho_{2}$ are the density jumps.

Since our study concerns only the cases of the upperand middle-layer monopole propagation, we set the lowerlayer potential vorticity to be always time-independent. Additionally, either the upper- or middle-layer potential vorticity has one time-dependent singular value moving with the monopole's centre. Hence, we have two sets of singular perturbations of the flow:

$$
\begin{gathered}
q_{m}=q_{m}^{*}+\frac{f}{H_{m}} \mu_{m} \delta\left(\left|\boldsymbol{r}_{i}-\boldsymbol{r}_{m}^{*}\right|\right), \\
q_{3-m}=q_{3-m}^{*}, \quad q_{3}=q_{3}^{*},
\end{gathered}
$$

where $m=1$ corresponds to the upper-layer monopole case, $m=2$ corresponds to the middle-layer monopole case, $\mu_{m}$ is the monopole's strength and $\boldsymbol{r}_{m}^{*}$ is the position of the monopole's singularity within the $m$-layer, $q_{i}^{*}$ is the potential vorticity background value, and $\left|\boldsymbol{r}_{i}-\boldsymbol{r}_{m}^{*}\right|=$ $\sqrt{\left(x_{i}-x_{m}^{*}\right)^{2}+\left(y_{i}-y_{m}^{*}\right)^{2}}$ with $x_{i}, y_{i}$ being Cartesian coordinates of a fluid particle within the $i$-layer.

Potential vorticities (Eq. A1) should satisfy the potential vorticity conservation law in each layer,

$\partial_{t} q_{i}+J\left(\psi_{i}, q_{i}\right)=0$

To obtain explicit analytical relations for stream functions $\psi_{i}$, one can split relations (Eq. A1) (see e.g. Gryanik and Tevs, 1989). Omitting intermediate transformations, we explicitly obtain barotropic mode $\Phi_{1}$ and two baroclinic modes $\Phi_{2}, \Phi_{3}$ for the upper $(m=1)$ and middle $(m=2)$ layer 


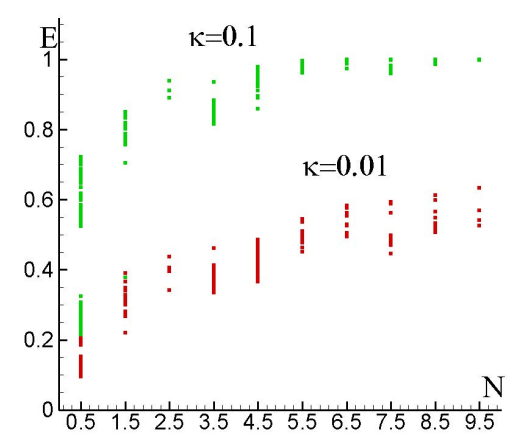

(a) upper-layer positive $\kappa$ monopole

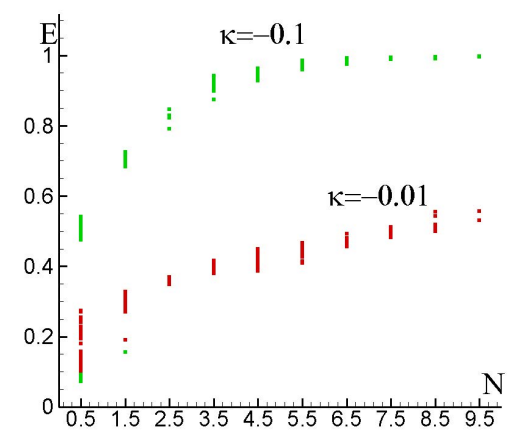

(b) upper-layer negative $\kappa$ monopole

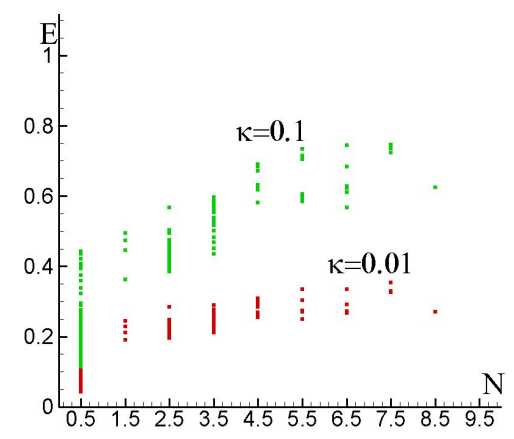

(c) middle-layer positive $\kappa$ monopole

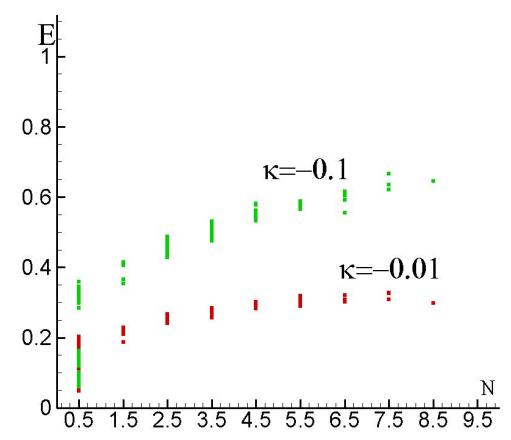

(d) middle-layer negative $\kappa$ monopole

Fig. 13. Lagrangian advection efficiency $E$ depending on monopole revolution number $N$. monopole cases

$$
\begin{aligned}
& \Phi_{1 m}=\frac{f}{\gamma}\left(\frac{(-1)^{3-m}\left(\alpha_{3-m}-\beta_{3-m}\right) \mu_{1}}{H_{1}} \log \left(r_{i 1}^{*}\right)+\right. \\
& \left.+\frac{\left(\alpha_{1} \beta_{2}-\alpha_{2} \beta_{1}\right) \tau}{H_{3}} \log \left(r_{i}\right)\right), \\
& \Phi_{2 m}=-\frac{f}{\gamma}\left(\frac{(-1)^{3-m}\left(\beta_{3-m}-1\right) \mu_{1}}{H_{1}} K_{0}\left(\sqrt{k_{3}\left(\alpha_{2}-1\right)} r_{i 1}^{*}\right)+\right. \\
& \left.+\frac{\left(\beta_{1}-\beta_{2}\right) \tau}{H_{3}} K_{0}\left(\sqrt{k_{3}\left(\alpha_{2}-1\right)} r_{i}\right)\right), \\
& \Phi_{3 m}=-\frac{f}{\gamma}\left(\frac{(-1)^{3-m}\left(1-\alpha_{3-m}\right) \mu_{1}}{H_{1}} K_{0}\left(\sqrt{k_{3}\left(\beta_{2}-1\right)} r_{i 1}^{*}\right)+\right. \\
& \left.+\frac{\left(\alpha_{2}-\alpha_{1}\right) \tau}{H_{3}} K_{0}\left(\sqrt{k_{3}\left(\beta_{2}-1\right)} r_{i}\right)\right),
\end{aligned}
$$

Now, introducing a nonvortical plane boundary source flux in the form $-U y$, which generates no vorticity and is compensated by an analogous drain flux (e.g. Izrailsky et al., 2004), where $U$ is a characteristic velocity, the final stream functions of the three-layer model with the monopole moving within the $m$-layer are

$\psi_{i m}=-U y+\Phi_{1 m}+\alpha_{i} \Phi_{2 m}+\beta_{i} \Phi_{3 m}$,

where $\alpha_{3}=\beta_{3}=1$.

\section{Supplementary material related to this article is available online at: \\ http://www.nonlin-processes-geophys.net/20/107/2013/ npg-20-107-2013-supplement.zip.}

Acknowledgements. The reported study was partially supported by RFBR, research projects: 11-05-00025-A, 12-05-31011; by the Ministry of Education and Science of Russia, project: 14.A18.21.0353; and by the ICMAT Severo Ochoa project SEV2011-0087. We also thank the anonymous reviewers for their useful comments on the paper.

Edited by: A. M. Mancho

Reviewed by: two anonymous referees 


\section{References}

An, B. W. and McDonald, N. R.: Coastal currents and eddies and their interaction with topography, Dyn. Atmos. Oceans, 40, 237253, 2005.

Aref, H.: Stirring by chaotic advection, J. Fluid Mech., 143, 1-21, 1984.

Aref, H.: The development of chaotic advection, Phys. Fluids, 14, 1315-25, 2002.

Aref, H. and Brons, M.: On stagnation points and streamline topology in vortex flows, J. Fluid Mech., 370, 1-27, 1998.

Baines, P. G.: Topographic effects in stratified flows, Cambridge University Press, 1993.

Baines, P. G. and Smith, R. B.: Upstream stagnation points in stratified flow past obstacles, Dyn. Atmos. Oceans, 18, 105-113, 1993.

Branicki, M. and Wiggins, S.: Finite-time Lagrangian transport analysis: stable and unstable manifolds of hyperbolic trajectories and finite-time Lyapunov exponents, Nonlin. Processes Geophys., 17, 1-36, doi:10.5194/npg-17-1-2010, 2010.

Candon, S. and Marshall, J. S.: Vortex ring deformation, capture, and entrainment by a columnar vortex, Phys. Fluids, 24, 093604, doi:10.1063/1.4753946, 2012.

Carton, X.: Hydrodynamical modeling of oceanic vortices, Surv. Geophys., 22, 179-263, 2001.

Carton, X., Chérubin, L., Paillet, J., Morel, Y., Serpette, A., and Cann, B. L.: Meddy coupling with a deep cyclone in the Gulf of Cadiz, J. Mar. Syst., 32, 13-42, 2002.

Chelton, D. B., Schlax, M. G., Samelson, R. M., and de Szoeke, R. A.: Global observations of large oceanic eddies, Geophys. Res. Lett., 34, L15606, doi:10.1029/2007GL030812, 2007.

Chelton, D. B., Schlax, M. G., and Samelson, R. M.: Global observations of nonlinear mesoscale eddies, Prog. Oceanogr., 91, 167-216, 2011.

Dewar, W. K.: Baroclinic eddy interaction with isolated topography, J. Phys. Oceanogr., 32, 2789-2805, 2002.

Filyushkin, B. N. and Sokolovskiy, M. A.: Modeling the evolution of intrathermocline lenses in the Atlantic Ocean, J. Mar. Res., 69, 191-220, 2011.

Filyushkin, B. N., Sokolovskiy, M. A., Kozhelupova, N. G., and Vagina, I. M.: Reflection of Intrathermocline Eddies on the Ocean Surface, Doklady Earth Sci., 439, 986-989, 2011.

Gryanik, V. M.: Dynamics of singular geostrophical vortices in a 2level model of the atmosphere (ocean), Izvestiya, Atmos. Ocean. Phys., 19, 227-240, 1983.

Gryanik, V. M. and Tevs, M. V.: Dynamics of singular geostrophical vortices in a N-level model of the atmosphere (ocean), Izv., Atmos. Ocean. Phys., 25, 179-188, 1989.

Gryanik, V. M., Doronina, T. N., Olbers, D. J., and Warncke, T. H.: The theory of three-dimensional hetons and vortex-dominated spreading in localized turbulent convection in a fast rotating stratified fluid, J. Fluid Mech., 423, 71-125, 2000.

Herbette, S., Morel, Y., and Arhan, M.: Erosion of a surface vortex by a seamount, J. Phys. Oceanogr., 33, 1664-1679, 2003.

Herbette, S., Morel, Y., and Arhan, M.: Erosion of a surface vortex by a seamount on the beta plane, J. Phys. Oceanogr., 35, 20122030, 2005.

Izrailsky, Y. G., Kozlov, V. F., and Koshel, K. V.: Some specific features of chaotization of the pulsating barotropic flow over elliptic and axisymmetric sea-mounts, Phys. Fluids, 16, 3173-3190,
2004.

Izrailsky, Y. G., Koshel, K. V., and Stepanov, D. V.: Determination of the optimal excitation frequency range in background flows, CHAOS, 18, 013107, doi:10.1063/1.2835349, 2008.

Koshel, K. V. and Prants, S. V.: Chaotic advection in the ocean, Physics-Uspekhi, 176, 1177-1206, 2006.

Koshel, K. V., Sokolovskiy, M. A., and Davies, P. A.: Chaotic advection and nonlinear resonances in an oceanic flow above submerged obstacle, Fluid Dyn. Res., 40, 695-736, 2008.

Kozlov, V. F.: Background currents in geophysical hydrodynamics, Izv. Atmos. Ocean. Phys., 31, 245-250, 1995.

Kozlov, V. F. and Koshel, K. V.: Barotropic model of chaotic advection in background flows, Izv. Atmos. Ocean. Phys., 35, 638648, 1999.

Kozlov, V. F. and Koshel, K. V.: A model of chaotic transport in the barotropic background flow, Izv. Atmos. Ocean. Phys., 36, 119-128, 2000.

Kozlov, V. F. and Koshel, K. V.: Some features of chaos development in an oscillatory barotropic flow over an axisymmetric submerged obstacle, Izv. Atmos. Ocean. Phys., 37, 351-361, 2001.

Kozlov, V. F., Koshel, K. V., and Stepanov, D. V.: Influence of the boundary on chaotic advection in the simplest model of a topographic vortex, Izv. Atmos. Ocean. Phys., 41, 217-227, 2005.

Lichtenberg, A. and Lieberman, M.: Regular and Stochastic Motion, Springer-Verlag, New York, 1983.

Mancho, A. M., Small, D., and Wiggins, S.: A tutorial on dynamical systems concepts applied to Lagrangian transport in oceanic flows defined as finite time data sets: Theoretical and computational issues, Phys. Rep., 437, 55-124, 2006.

Mendoza, C., Mancho, A. M., and Rio, M.-H.: The turnstile mechanism across the Kuroshio current: analysis of dynamics in altimeter velocity fields, Nonlin. Processes Geophys., 17, 103-111, doi:10.5194/npg-17-103-2010, 2010.

Pedlosky, J.: Geophysical Fluid Dynamics, 2nd Edn., Springer, New York, 1987.

Reznik, G. M.: Dynamics of localized vortices on the beta plane, Izv. Atmos. Ocean. Phys., 46, 784-797, 2010.

Reznik, G. M. and Kizner, Z.: Two-layer quasi-geostrophic singular vortices embedded in a regular flow, Part 1. Invariants of motion and stability of vortex pairs, J. Fluid Mech., 584, 185-202, 2007a.

Reznik, G. M. and Kizner, Z.: Two-layer quasi-geostrophic singular vortices embedded in a regular flow, Part 2. Steady and unsteady drift of individual vortices on a beta-plane, J. Fluid Mech., 584, 185-202, 2007b.

Reznik, G. M. and Kizner, Z.: Singular vortices in regular flows, Theor. Comput. Fluid Dyn., 24, 65-75, 2010.

Rypina, I. I., Scott, S. E., Pratt, L. J., and Brown, M. G.: Investigating the connection between complexity of isolated trajectories and Lagrangian coherent structures, Nonlin. Processes Geophys., 18, 977-987, doi:10.5194/npg-18-977-2011, 2011.

Ryzhov, E. A.: On changing the size of the atmosphere of a vortex pair embedded in a periodic external shear flow, Phys. Lett. A, 375, 3884-3889, 2011.

Ryzhov, E. A. and Koshel, K. V.: The Effects of Chaotic Advection in a Three-Layer Ocean Model, Izv. Atmos. Ocean. Phys., 47, 241-251, 2011a.

Ryzhov, E. A. and Koshel, K. V.: Estimating the size of the regular region of a topographically trapped vortex, Geophys. Astrophys. 
Fluid Dyn., 105, 536-551, 2011 b.

Ryzhov, E. A., Koshel, K. V., and Carton, X. J.: Passive scalar advection in the vicinity of two point vortices in a deformation flow, Eur. J. Mech. B- Fluid., 34, 121-130, 2012.

Sokolovskiy, M. A.: Stability of an axisymmetric three-layer vortex, Izv. Atmos. Ocean. Phys., 33, 19-30, 1997.

Sokolovskiy, M. A., Zyryanov, V. N., and Davies, P. A.: On the influence of an isolated submerged obstacle on a barotropic tidal flow, Geophys. Astrophys. Fluid Dyn., 88, 1-30, 1998.

Sutyrin, G., Herbette, S., and Carton, X.: Deformation and splitting of baroclinic eddies encountering a tall seamount, Geophys. Astrophys. Fluid Dyn., 105, 478-505, 2011.

Titaud, O., Brankart, J., and Verron, J.: On the use of Finite-Time Lyapunov Exponents and Vectors for direct assimilation of tracer images into ocean models, Tellus, 63A, 1038-1051, 2011.
Voropayev, S. I., Smirnov, S. A., and Brandt, A.: Dipolar eddies in a stratified shear flow, Phys. Fluids, 13, 3820, doi:10.1063/1.1416499, 2001.

van Geffen, J. H. G. M. and Davies, P. A.: Interaction of a monopolar vortex with a topographic ridge, Geophys. Astrophys. Fluid Dyn., 90, 1-41, 1999.

Wang, G. H. and Dewar, W. K.: Meddy-seamount interactions: Implications for the Mediterranean salt tongue, J. Phys. Oceanogr., 33, 2446-2461, 2003.

Wiggins, S.: Chaotic Transport in Dynamical Systems, SpringerVerlag, New York, 1992.

Zaslavsky, G.: Physics of Chaos in Hamiltonian Dynamics, Imperial College Press, London, 1998.

Zavala Sansón, L., Aguiar, A. C. B., and van Heijst, G. J. F.: Horizontal and vertical motions of barotropic vortices over a submarine mountain, J. Fluid Mech., 695, 173-198, 2012. 